\title{
Screening of Extended-Spectrum $\beta$-Lactamases (ESBL)-producing Klebsiella pneumoniae with ChromID ESBL Media
}

\author{
Emy Noerwidayati, Andaru Dahesihdewi, Osman Sianipar* \\ Department of Clinical Pathology and Laboratory Medicine, Faculty of Medicine, Universitas Gadjah Mada, \\ Jl. Farmako Sekip Utara, Yogyakarta 55284, Indonesia \\ *Corresponding author. E-mail: osmansianipar@ugm.ac.id
}

Received date: Jan 23, 2018; Revised date: May 30, 2018; Accepted date: Jun 5, 2018

\section{Abstract}

$\mathrm{B}$

ACKGROUND: Klepsiella pneumoniae, one of clinical isolates, is frequently found causative agent of hospital acquired infection. Currently, $K$. pneumoniae is found as extended-Spectrum $\beta$-lactamases (ESBL) producer, allowing it to become multidrugresistant. A clinical laboratory with limited facility needs a valid, reliable, inexpensive and simple laboratory test to control its infection and antimicrobial-resistancy. The aim of this study is to evaluate the diagnostic performance of a ESBL media to detect ESBL-producing K. pneumoniae.

METHODS: An independent and blind comparative study of ChromID ESBL media and Double Disc Synergy Test (DDST) was conducted for detecting the clinical isolate of ESBL-producing K. pneumoniae. Clinical isolates of $K$. pneumoniae collected from the Clinical Laboratory of Dr. Sardjito Hospital were isolated.
RESULTS: There were 103 clinical isolates of $K$. pneumoniae, which were isolated from urine, pus, blood, stool, cerebrospinal fluid, sputum, drain liquid, nasal sinus liquid, gastric wash, bronchi liquid, injury liquid and nasal swab. The number of true positive, true negative, false positive and false negative results were 74, 18, 9 and 2, respectively. Meanwhile, the sensitivity, specificity, positive predictive value, negative predictive value, likelihood ratio for positive result and likelihood ratio for negative result of the new ESBL media were $97.4 \%, 66.7 \%, 89.2 \%, 90 \%, 2.9$ and 0.03 , respectively.

CONCLUSION: Since the new ESBL media and DDST results were similar, so the new ESBL media could be used for screening patients with clinical presentation that indicating a high suspicious of ESBL-producing bacteria.

KEYWORDS: K. pneumoniae, ChromID ESBL, DDST, ESBL, sensitivity

Indones Biomed J. 2018; 10(3): 217-21

\section{Introduction}

In developing countries, infectious diseases remain as the first cause of illness in community. Treatments for infections usually involve antibiotics. With many advances in technology, the number and type of clinically useful antibiotics is increasing. Inappropriate antibiotic treatment may result adverse effects, such as the emergence of bacterial resistance and low antibiotic efficacy against certain bacteria. Bacterial resistance against antibiotics are a current global issue.(1)

Resistance against antibiotics is mostly caused by nosocomial infections. Klebsiella spp. are important pathogenic bacteria causing nosocomial infections. Generally, Klebsiella spp. infections are implicated in pneumonia, urinary tractus infections, meningitis and septicemia. The increasing resistancy of Klebsiella spp. has been widely reported as the effect of enzymes extended-spectrum $\beta$-lactamases (ESBL) and Klebsiella 
pneumoniae carbapenemase (KCP).(2) ESBL is an enzyme that hydrolyze penicillin, cephalosporin (first, second, third generations), oxymino- $\beta$-lactam compound (aztreonam, but not cephamycin and carbapenem) and may be inhibited by $\beta$-lactamase inhibitor (clavulanate, sulbactam and tazobactam).(3)

Various screening and confirmation tests have been developed to detect ESBL-producing $K$. pneumoniae. However, the increasing diversity of ESBL-producing $K$. pneumoniae strains, along with resistance mechanism of non-ESBL-producing K. pneumoniae, making identification of ESBL-producing $K$. pneumoniae by conventional phenotypic techniques difficult.(4) Double Disc Synergy Test (DDST) is able to find the phenotypic character profile of ESBL resistance against third generation cephalosporin (ceftazidim, cefpodoxime, ceftriaxone) and monobactams (aztreonam). The presence of ESBL-producing bacteria was indicated by an increase in inhibitory zone on amoxicillin/ clavulanic acid through third-generation cephalosporins.(5)

There was a breakthrough in the development of selective chromogenic medium for the screening of ESBL-producing $K$. pneumoniae directly from clinical specimens. Selectivity was provided based on colony color by chromogenic media.(6) New ESBL media with chromogenic medium has been developed. It contains nutrient rich media with a mixture of antibiotics, including cefpodoxime for detection of ESBL-bearing bacteria.(7) A study reported that the new ESBL media had a sensitivity of $88 \%$ and specificity of $94.4 \%$ in detecting ESBL-producing Enterobactericeae in 24 hours.(8)

In detecting antimicrobial resistance, the role of routine laboratory services is very essential. Surveillance of bacterial pattern, antibiogram pattern, multiresistant bacterial detection is an important support for the treatment of patients with infections and reasonable antibiotic prescription. The objective of the study is to evaluate the sensitivity of new ESBL media with chromogenic medium in detecting ESBL-producing K. pneumoniae.

\section{Methods}

A diagnostic test study using 103 clinical isolates of $K$. pneumoniae was conducted. In this study, the ChromID® ESBL media (bioMérieux, Marcy-l'Étoile, France) was used as the new ESBL media with chromogenic medium. The ESBL media was independently and blindly compared to the DDST, the gold standard test in detecting ESBLproducing $K$. pneumoniae. DDST is known as one of the confirmatory test (9) and has sensitivity of $91.3 \%$ and specificity of $100 \%$.(10)

The clinical isolates of $K$. pneumoniae were obtained from urine, pus, blood, feces, sputum, cerebrospinal fluid, drain liquid, nasal sinus liquid, gastric wash, bronchi liquid, injury liquid and nasal swab. The clinical samples were inoculated on the appropriate media. After the growth was detected, identification of $K$. pneumoniae was performed using Vitek 2 System (bioMérieux).

The identified isolates of $K$. pneumoniae were inoculated on the ESBL media and incubated at $35^{\circ} \mathrm{C}$ for 18-24 hours, followed by observation and interpration. The growth of green colonies were interpretated as ESBLproducing $K$. pneumoniae.

DDST was performed by inoculating 0.5 McFarland turbidity standard of $K$. pneumoniae suspension on the Mueller Hinton solid media plate. After the suspension was dried, $30 \mu \mathrm{g}$ ceftazidim, $30 \mu \mathrm{g}$ cefotaxime, $30 \mu \mathrm{g}$ cefepime and 20/10 $\mu \mathrm{g}$ amoxycillin/clavulanic acid disc were added on the media. The media plates were incubated at $35^{\circ} \mathrm{C}$ for 18 24 hours. The presence/absence of an increase in inhibitory zone between $\beta$-lactam discs and $\beta$-lactam $/ \beta$-lactamase inhibitor disc can be observed after the incubation.

K. pneumoniae ATCC 7000603 was used as the positive control of ESBL-producing bacteria, whereas Escherichia coli ATCC 25922 was used as the negative control of non-ESBL-producing bacteria. The results of ESBL media and DDST were intepreted by two observers.

The collected data were analyzed using decriptive statistic. Diagnostic performance was evaluated using $2 \times 2$ table. The sensitivity, specificity, accuracy, positive predictive value, negative predictive value, positive likelihood ratio and negative likelihood ratio were determined with $95 \%$ confidence interval.

The study was conducted in Clinical Laboratory of Dr. Sardjito Hospital Yogyakarta, Indonesia started from July to September 2015. Ethical clearance was issued by the Medical and Health Research Ethics Committee Faculty of Medicine, University of Gadjah Mada, Yogyakarta, Indonesia No. KE/KF/868/EC/2015.

\section{Results}

Total number of K. pneumoniae isolates were 103, collected from patients with different ages, as shown in Table 1. Sources of isolates were mostly urine $(29.1 \%)$, pus $(28.2 \%)$ and blood (22.3\%). Urine specimens were collected from patients diagnosed with urinary tractus infection, chronic 
Table 1. Characteristics of sources of clinical isolates.

\begin{tabular}{|c|c|c|}
\hline Characteristics of isolates & $\mathbf{n}$ & $\%$ \\
\hline Number of isolates & 103 & 100 \\
\hline \multicolumn{3}{|l|}{ Age } \\
\hline Toddlers ( $<5$ years) & 34 & 33 \\
\hline Child ( $\geq 5-<18$ years) & 10 & 9.7 \\
\hline Adults ( $\geq 18-<55$ years) & 32 & 31.1 \\
\hline Elderly ( $\geq 55$ years) & 27 & 26.2 \\
\hline \multicolumn{3}{|l|}{ Source of isolates } \\
\hline Urine & 30 & 29.1 \\
\hline Pus & 29 & 28.2 \\
\hline Blood & 23 & 22.3 \\
\hline Feces & 11 & 10.7 \\
\hline Cerebrospinal fluid & 2 & 1.9 \\
\hline Sputum & 1 & 1.0 \\
\hline Stool & 1 & 1.0 \\
\hline Drain liquid & 1 & 1.0 \\
\hline Nasal sinus liquid & 1 & 1.0 \\
\hline Gastric wash & 1 & 1.0 \\
\hline Bronchi liquid & 1 & 1.0 \\
\hline Injury liquid & 1 & 1.0 \\
\hline Nasal swab & 1 & 1.0 \\
\hline \multicolumn{3}{|l|}{ Types of clinical care } \\
\hline Intensive & 19 & 18.5 \\
\hline Non-intensive: - Surgical & 9 & 8.7 \\
\hline - Non Surgical & 61 & 59.2 \\
\hline Outpatient & 14 & 13.6 \\
\hline
\end{tabular}

renal failure, acute renal failure and kidney stones. Pus specimens were collected from patients diagnosed with diabetic ulcers, mandibula abscess, post laparotomy, colon abscess and nasal cavity carcinoma. The patients were mostly from non-intensive care without surgical treatment (59.2\%). Meanwhile, $18.5 \%$ of patients were taken care in intensive care unit. Detail characteristics of isolates regarding age, source of isolates and their respective ward were shown in Table 1.
Result of new ESBL media and DDST were shown in Table 2. DDST was able to detect 76 isolates of ESBLproducing K. pneumoniae (73.8\%), whereas the ChromID ${ }^{\circledR}$ ESBL media was able to detect 83 isolates of ESBLproducing K. pneumoniae (80.6\%). By using DDST as the standard, there were 9 samples with false positive and 2 samples with false negative results of new ESBL media.

Table 3 showed the test characteristic of ChromID ESBL media in detecting the ESBL-producing $K$. pneumoniae. New ESBL media had high sensitivity (97.4\%). The media also had positive $(89.2 \%)$ and negative $(90 \%)$ predictive value.

\section{Discussion}

In this study, study subjects catagorized as elderly ( $\geq 55$ years old) was $26.2 \%$. Meanwhile previous study in hemodialysis patients whom suffered from bacteremia caused by $K$. pneumoniae reported that the proportion of patients older than 65 years old was $57.8 \%$.(11) Another study reported that majority of study subjects (54.2\%) whom suffered from $K$. pneumoniae bloodstream infections were aged more than 60 years.(12)

Most of subjects in this study (68\%) were from nonintensive care unit and only $18.5 \%$ were from the intensive care unit. A study in hemodialysis patients who also suffered from $K$. pneumoniae bacteremia, reported that $46.7 \%$ of subjects were from the intensive care unit. Another study reported that $67.9 \%$ of hospital-acquired $K$. pneumoniae infections were particularly among patients from neonatal intensive care unit, with mortality rate up to $70 \%$.(13)

Reglier-Poupet stated that longer incubation period of new ESBL media test may improve the sensitivity of this test. Negative results obtained from the new ESBL media can be improved with longer incubation. Incubation for 24 hours was associated with sensitivity of $88 \%$ and specificity of $94.4 \%$. Meanwhile, incubation for 48 hours resulted in sensitivity of $94 \%$ and specificity of $90.5 \%$. In the new ESBL media test with a reincubation period for 48 hours, colonies still did not grow, indicating no ESBL-producing

Table 2. Comparison between ChromID ESBL media and DDST.

\begin{tabular}{llccc}
\hline & & \multicolumn{2}{c}{ DDST } & \multirow{2}{*}{ Total } \\
\cline { 3 - 4 } & & ESBL (+) & ESBL (-) & \\
\hline ChromID ® ESBL Test & ESBL (+) & 74 & 9 & 83 \\
& ESBL (-) & 2 & 18 & 20 \\
Total & & 76 & 27 & 103 \\
\hline
\end{tabular}


Table 3. Test characteristic of ChromID ESBL.

\begin{tabular}{lcc}
\hline & Value & $\mathbf{9 5 \%}$ CI \\
\hline Sensitivity (\%) & 97.4 & $90.82-99.68$ \\
Specificity (\%) & 66.7 & $46.04-83.48$ \\
Positive Predictive Value (\%) & 89.2 & $80.35-94.95$ \\
Negative predictive value (\%) & 90 & $68.30-98.77$ \\
Positive Likelihood Ratio & 2.9 & $1.71-4.99$ \\
Negative Likelihood Ratio & 0.03 & $0.01-0.16$ \\
\hline
\end{tabular}

isolate.(8) In our current results, there were 2 samples with false negative result, although the media had been incubated for 48 hours. The colonies still did not grow, indicating no ESBL-producing isolate.(8) In this study, longer incubation period (reincubation) were not performed for false negative results. In these falsenegative results, patients actually had the disease, but results showed non-ESBL-producing $K$. pneumoniae.

In this study, there were 9 false positive results. These false positive results might be related with the antibiotic used in the new ESBL media test. There was only one type of antibiotic, cefpodoxime, while in DDST there were combinations of antibiotics including cefotaxime, amoxicilin, clavulanic acid. Therefore, false positive results could happened, whereas, shown as resistant results. The majority of ESBL enzymes, are different significantly in biochemical characteristics such as activity against specific $\beta$-lactam (e.g., cefotaxime, ceftazidime).(14) In false positive results, possibly there were non-Enterobacteriaceae isolates, Enterobacteriaceae strains with sefalosporinase, or penicillinase (Klebsiella oxytoca). The difficulty of the new ESBL media was to distinguish between ESBL-producing isolates and isolates that associated with other resistant mechanisms, i.e., strain mediated AmpC $\beta$-lactamase over production and strain with over production of $\beta$-lactamase in K. oxytoca.(8)

The new ESBL media test could be used for screening patients with clinical presentation that indicating a high suspicious of ESBL-producing bacteria. The new ESBL media could be an appropriate method for monitoring critically ill patients with ESBL carrier. Low specificity in this study, suggested that all suspected ESBL-producing bacteria that were tested with the new ESBL media should be confirmed with a confirmation test, for example DDST.(15)

In current study, this new ESBL media showed sensitivity of $97.4 \%$ and specificity of $66.7 \%$. Another study reported that the new ESBL meda test had sensitivity of $94.9 \%$ and specificity of $94.9 \%$ in detecting ESBLproducing Enterobactericeae.(16)

\section{Conclusion}

The new ESBL media and DDST results were similar. The sensitivity, specificity, positive predictive value and negative predictive value of the new ESBL media were appropriate. Therefore, it could be used for for screening patients with clinical presentation that indicating a high suspicious of ESBL-producing bacteria. All suspected ESBL-producing bacteria that were tested with the new ESBL media should be confirmed with a confirmation test, such as DDST.

\section{Acknowledgement}

We would like to thank the Director of Dr. Sardjito Hospital who has given permission to conduct this study. Our gratitude also goes to the Chair of The Department of Clinical Pathology and Laboratory Medicine, to the Chair of Clinical Laboratory of Dr. Sardjito Hospital, together with the staffs whom supported the study, as well as to the patients who have been willing to participate as the research subjects.

\section{References}

1. Roca I, Akova M, Baquero F, Carlet J, Cavaleri M, Coenen S, et al. The global threat of antimicrobial resistance :science for intervention. New Microbes New Infect 2015; 6: 22-9.

2 Lee C, Lee JH, Park KS, Kim YB, Jeong BC, Lee SH. Global dissemination of Klebsiella pneumoniae : epidemiology, genetic context, treatment options, and detection methods. Front Microbiol. 2016; 7: 895. doi: 10.3389/fmicb.2016.00895.

3 Ghafourian S, Sadeghifard N, Soheili S, Sekawi Z. Extended spectrum beta-lactamases. Curr Issues Mol Biol. 2015; 17: 11-22.

4 Subashini J, Kannabiran K. Screening and identification of extended spectrum $\beta$-lactamase (ESBL) pathogens in urine sample of UTI Patient's. Trop Med Surg. 2013; 1: 120. doi: 10.4172/23299088.1000120.

5 Numanovic F, Hukic M, Delibegovic Z, Tihic N, Pasic S, Gegic M. Comparison of double disk synergy test, VITEK 2 and Check-MDR CT102 for detection of ESBL producing isolates. Acta Med Acad. 2013; 42: 15-24.

6 Diederen BMW, Leest M Van, Van I, Willemse P, Keulen PHJ Van, Jan AJW, et al. Performance of MRSA ID, a new chromogenic medium for detection of methicillin-resistant Staphylococcus aureus. J Clin Microbiol. 2006 Feb; 44(2): 586-588.

7 Pfaller MA, Segreti J. Overview of the epidemiological profileand laboratory detection of extended-spectrum $\beta$-Lactamases. Clin Infect Dis. 2006; 42: S153-63. 
8 Réglier-Poupet H, Naas T, Carrer A, Cady A, Adam JM, Fortineau $\mathrm{N}$, et al. Performance of chromID ESBL, a chromogenic medium for detection of Enterobacteriaceae producing extended-spectrum B-lactamases. J Med Microbiol. 2008; 57: 310-5.

9 Clinical Laboratory Standards Institute. M100S Performance Standards for Antimicrobial. Wayne: Clinical and Laboratory Standards Institute; 2016.

10 Wiegand I, Geiss HK, Mack D, Stürenburg E, Seifert H. Detection of extended-spectrum beta-lactamases among Enterobacteriaceae by use of semiautomated microbiology systems and manual detection procedures. J Clin Microbiol. 2007; 45: 1167-74.

11 Harada S, Ishii Y, Yamaguchi K. Extended-spectrum $\beta$-Lactamases: implications for the clinical laboratory and therapy. Korean J Lab Med. 2008; 28: 401-12.

12 Yang $\mathrm{CC}$, Wu CH, Lee CT, Liu HT, Chen JB, Chiu CH, et al. Nosocomial extended-spectrum beta-lactamase-producing Klebsiella pneumoniae bacteremia in hemodialysis patients and the implications for antibiotic therapy. Int J Infect Dis. 2014; 28: 3-7.

13 Liu YM, Li BB, Zhang YY, Zhang W, Shen H, Li H, et al. Clinical and molecular characteristics of emerging hypervirulent Klebsiella pneumoniae bloodstream infections in mainland China. Antimicrob Agents Chemother 2014; 58: 5379-85

14 Gupta A, Ampofo K, Rubenstein D, Saiman L. Extended spectrum $\beta$ lactamase-producing Klebsiella pneumoniae infections: A review of the literature. J Perinatol. 2003; 23: 439-43.

15 Färber J, Moder KA, Layer F, Tammer I, König W, König B. Extended-spectrum beta-lactamase detection with different panels for automated susceptibility testing and with a chromogenic medium. J Clin Microbiol. 2008; 46: 3721-7.

16 Huang T, Bogaerts P, Berhin C, Guisset A, Glupczynski Y. Evaluation of brilliance ESBL agar, a novel chromogenic medium for detection of extended-spectrum-beta-lactamase-producing enterobacteriaceae. 2010; 48: 2091-6. 